\title{
Inhibition of MicroRNA-96 Ameliorates Cognitive Impairment and Inactivation Autophagy Following Chronic Cerebral Hypoperfusion in the Rat
}

\author{
Peifang Liu ${ }^{a}$ Peijia Liu ${ }^{b}$ Zhiyong Wang ${ }^{c}$ Shaohong Fangd,e Yuting Liu ${ }^{f}$ \\ Jinhua Wang ${ }^{g}$ Wenjuan Liu ${ }^{a}$ Ning Wang $^{\text {a }}$ Lixia Chen $^{\mathrm{a}}$ Jianjian Wang ${ }^{\mathrm{a}}$ \\ Huixue Zhang ${ }^{\text {a }}$ Lihua Wang ${ }^{\text {a }}$
}

\begin{abstract}
aDepartment of Neurology, The Second Affiliated Hospital of Harbin Medical University, Harbin, Heilongjiang Province, ${ }^{b}$ Department of Clinical Laboratory, The Second Affiliated Hospital of Harbin Medical University, Harbin, Heilongjiang Province, 'Ministry of Education of Harbin Medical University, Harbin, Heilongjiang Province, dDepartment of Cardiology, Harbin Medical University, Ministry of Education, Heilongjiang Province, eThe Key Laboratory of Myocardial Ischemia, Harbin Medical University, Ministry of Education, Harbin, Heilongjiang Province, ${ }^{f}$ Department of Nuclear Medicine, The Second Affiliated Hospital of Harbin Medical University, Harbin, Heilongjiang Province, ${ }^{9}$ Department of Pharmacy Intravenous Admixture Services, The First Affiliated Hospital of Harbin Medical University, Harbin, Heilongjiang Province, China
\end{abstract}

\section{Key Words}

MicroRNA • Autophagy $•$ Chronic cerebral hypoperfusion $\bullet$ MTOR $\bullet$ LC3

\begin{abstract}
Background/Aims: Chronic cerebral hypoperfusion $(\mathrm{CCH})$ is a high-risk factor for vascular dementia and Alzheimer's disease. Autophagy plays a critical role in the initiation and progression of $\mathrm{CCH}$. However, the underlying mechanisms remain unclear. In this study, we identified the effect of a microRNA (miR) on autophagy under $\mathrm{CCH}$. Methods: A $\mathrm{CCH}$ rat model was established by two-vessel occlusion (2VO). Learning and memory abilities were assessed by the Morris water maze. The protein levels of LC3, beclin-1, and mTOR were detected by western blotting and immunofluorescence assays, miR-96 expression was assessed by real-time PCR, luciferase assays were used to determine the effect of miR-96 on the $3^{\prime}$ untranslated region (UTR) of MTOR, and the number of autophagosomes was examined by electron microscopy. Results: The level of miR-96 was significantly increased in $2 \mathrm{VO}$ rats, and inhibition of miR-96 ameliorated the cognitive impairment induced by $2 \mathrm{VO}$. Furthermore, the number of LC3- and beclin-1-positive autophagosomes was increased in 2VO rats, and was decreased after miR-96 antagomir injection. However, the protein level of mTOR was P. Liu, P. Liu and Z. Wang contributed equally to this work.


reduced in $2 \mathrm{VO}$ rats, and it was down-regulated by miR-96 overexpression and up-regulated by miR-96 inhibition in $2 \mathrm{VO}$ rats and primary culture cells. Moreover, the luciferase activity of the 3'-UTR of mTOR was suppressed by miR-96, which was relieved by mutation of the miR-96 binding sites. Conclusion: Our study demonstrated that miR-96 may play a key role in autophagy under $\mathrm{CCH}$ by regulating mTOR; therefore, miR-96 may represent a potential therapeutic target for $\mathrm{CCH}$.

(C) 2018 The Author(s)

Published by S. Karger AG, Basel

\section{Introduction}

Senile dementia is a progressive cognitive impairment that is mainly classified as Alzheimer's disease (AD), vascular dementia (VaD), and other common types of dementia. $\mathrm{VaD}$ and $\mathrm{AD}$ often coexist in aged populations with dementia [1]. There is evidence that chronic cerebral hypoperfusion (CCH) contributes to the pathogenesis of both VaD and $A D$, which induces $A \beta$ aggregation, tau hyperphosphorylation, oxidative stress, synaptic dysfunction, and autophagy dysfunction [2,3].

Autophagy is an essential lysosomal degradation pathway that clears misfolded proteins and damaged organelles in cells $[4,5]$. In neurons, autophagy is responsible for the homeostatic pathway [6,7], and is also associated with $\mathrm{CCH}$ induced by two-vessel occlusion (2VO) surgery. For example, the accumulation of autophagic vacuoles and elevated microtubule-associated protein 1 light chain 3 (LC3) levels were found in the cortex of $2 \mathrm{VO}$ rats at 2 weeks after surgery [8]. Liu et al. reported that the level of LC3 was significantly increased in both the cortex and hippocampal CA1 area of $2 \mathrm{VO}$ rats at 5 weeks after surgery, and GABA receptors reversed the neuronal damage by activating autophagy [9]. These studies suggested that autophagy may play a critical role in $\mathrm{CCH}$-induced brain damage. However, the detailed mechanisms of CCH-induced autophagy dysfunction are not understood clearly.

MicroRNAs (miRNAs) are endogenous non-coding RNAs that regulate gene expression at the post-transcriptional level. Numerous studies have reported that miRNAs are involved in CCH-induced dementia. For example, miRNA (miR)-195 protects against dementia by regulating the expression of BACE 1 and APP and activating Cdk5/p25 and tau phosphorylation $[10,11]$. MiR-9 expression led to the dysfunction of Nav1.1 and Nav1.2 by targeting Nav $\beta 2$ in rats under $\mathrm{CCH}$ [12]. In addition, increasing evidence suggests an important role for miRNAs as mediators of autophagy. For example, miR-299-5p modulates autophagy and apoptosis by targeting ATG5, and miR-299-5p overexpression improves the cognitive impairment of APPswe/PS1dE9 mice [13]. CCH lead to upregulate the level of miR-27a at 2 weeks after $2 \mathrm{VO}$ surgery. MiR-27a induces dysfunction of the autophagic-lysosomal system by LAMP2, which is a key factor for autophagosome-lysosome fusion [14]. However, whether miR-96 participates in $\mathrm{CCH}$-induced autophagy dysfunction is not understood fully.

In this study, we established a $\mathrm{CCH}$ rat model induced by $2 \mathrm{VO}$ to examine whether the abnormal expression of miR-96 is associated with CCH-induced autophagy dysfunction at the post-transcriptional level, and to investigate the correlation between miR-96, autophagy, and cognitive impairment in $\mathrm{CCH}$.

\section{Materials and Methods}

\section{VO surgery}

Male Sprague-Dawley rats were obtained from the Animal Center at the Second Affiliated Hospital of Harbin Medical University and housed in a room with a constant humidity of $55 \pm 5 \%$. The CCH rat model was induced by $2 \mathrm{VO}$ surgery as described previously $[15,16]$. Briefly, after anesthetization, the common carotid arteries of the rats were exposed, and 5-0 silk sutures were used to perform permanent ligation. The rats were returned to their cages and were sacrificed at 2 months after 2 Vo surgery for further experiments. 


\section{Cellular Physiology Cell Physiol Biochem 2018;49:78-86 \begin{tabular}{ll|l} 
and Biochemistry Published onlıne: 22 August, 2018 & $\begin{array}{l}\text { (c) } 2018 \text { The Author(s). Published by S. Karger AG, Basel } \\
\text { www.karger.com/cpb }\end{array}$ \\
\hline
\end{tabular}}

Liu et al.: Microrna-96 Ameliorates Cognitive Impairment

\section{Stereotactic injection}

A stereotactic injection of miR-96 antagomir (Antagomir-96) was administered twice every 3 days starting at 1 week after 2VO surgery. Briefly, antagomir-96 was diluted in phosphate-buffered saline (PBS) and injected at sites $\mathrm{A}$ and $\mathrm{B}$, which were relative to the bregma (anteroposterior [AP], mediolateral [ML], and dorsoventral [DV]) as described previously [10]. Site A was AP, $-4.8 \mathrm{~mm}$; ML, $\pm 3.0 \mathrm{~mm}$; and DV, -3.5 $\mathrm{mm}$. Site B was AP, $-4.8 \mathrm{~mm}$; ML, $\pm 5.2 \mathrm{~mm}$; and DV, $-5.0 \mathrm{~mm}$. To avoid backflow, the needle was left for an additional 2 min after injection.

\section{Morris water maze}

A Morris water maze (MWM) hidden platform test was performed at 8 weeks after 2VO surgery as described previously $[17,18]$. Briefly, the maze was divided into 4 quadrants, and the 20 -cm diameter platform was located in the first quadrant. The maze was filled with opaque water by adding black nontoxic food pigment. Before training, the pupillary light reflex was tested, and disabled rats were excluded. On the first day, the rats were placed into the water facing the pool wall and left to find the hidden platform. The rats that did not find the platform within $120 \mathrm{~s}$ were guided by the researcher to the platform and allowed to rest on it for at least $20 \mathrm{~s}$. Over the next 4 days, the rats were allowed to find the hidden platform. The probe trial session was conducted for each rat on the sixth day. During this test, the hidden platform was removed from the first quadrant, and then the rats were allowed to swim freely from the third quadrant. Swim time was limited to $120 \mathrm{~s}$, and the number of rats crossing the first quadrant and the percentage of time spent swimming in the first quadrant were recorded and analyzed.

\section{Western blot analysis}

Proteins samples were extracted from the hippocampus of rats or primary neurons, which were lysed with RIPA buffer containing 1\% protease inhibitor cocktail. After measuring the protein concentration of the samples, the proteins were separated on a sodium dodecyl sulfate gel and then transferred onto a polyvinylidene fluoride membrane. After incubation in 5\% non-fat dry milk for $2 \mathrm{~h}$, the membrane was incubated with the primary antibodies for anti-LC3 (1:1000; L7543; Sigma, USA), anti-beclin-1 (1:200; sc11427; Santa Cruz Biotechnology, USA), anti-mammalian target of rapamycin (mTOR) (1:500; 2983; Cell Signaling Technology, USA), and $\beta$-actin (1:500; sc-47778; Santa Cruz Biotechnology, USA) at $4{ }^{\circ} \mathrm{C}$ overnight. The bands were captured on a BioSpectrum Imaging System and quantified with Quantity One software by normalizing to $\beta$-actin.

\section{Real-time RT-PCR analysis}

Total RNA was extracted from the hippocampus of rats or primary neurons using the TRIzol reagent (Invitrogen, Carlsbad, CA) according to manufacturer's instructions. Total RNA was reverse transcribed by SYBR $®$ Green Real-time PCR Master Mix (Toyobo, Osaka, Japan). The PCR was conducted as follows: $95{ }^{\circ} \mathrm{C}$ for $60 \mathrm{~s}$, followed by 40 cycles of $95^{\circ} \mathrm{C}$ for $15 \mathrm{~s}, 60{ }^{\circ} \mathrm{C}$ for $15 \mathrm{~s}$, and $72{ }^{\circ} \mathrm{C}$ for $45 \mathrm{~s}$. U6 was used as an internal control.

\section{Primary neuron culture}

The brains were removed from postnatal day 0 rat pups. After dissection and trituration, the cells were plated on 6-well plates pre-coated with poly-L-lysine $(0.1 \mathrm{mg} / \mathrm{mL})$ at a density of $2.0 \times 10^{6}$ cells/well. The cells were cultured in Neurobasal medium A with 2\% B27 supplement, $0.5 \mathrm{~mm}$ L-glutamine, $100 \mathrm{U} / \mathrm{mL}$ penicillin, and $100 \mathrm{mg} / \mathrm{mL}$ streptomycin The culture medium was changed every 3 days.

\section{Immunofluorescence analysis}

The prepared slices were fixed with $4 \%$ paraformaldehyde for 30 min. After blocking and permeabilization using PBS containing 10\% donkey serum and $0.1 \%$ Triton X-100 for $1 \mathrm{~h}$, the sections were incubated with an anti-LC3 primary antibody at $4{ }^{\circ} \mathrm{C}$ overnight. The sections were incubated with a secondary antibody conjugated to Alexa Fluor 594 (Invitrogen) for $1 \mathrm{~h}$. Images were captured using a confocal laser scanning microscope (FV 3000; Olympus, Tokyo, Japan). 
Luciferase assays

The 3 ' untranslated regions (UTRs) of wild-type and mutant mTOR were synthesized by Sangon Biotech Co., Ltd. HEK293-T cells were cultured and transfected with miR-96 mimics using Lipofectamine ${ }^{\text {TM }} 2000$ (Cat.no: 11668027, Invitrogen, USA) according to the manufacturer's instructions. After transfection for 48 $\mathrm{h}$, a dual luciferase reporter assay kit was used to detect luciferase activity according to the manufacturer's instructions.

\section{Statistical analysis}

All data are expressed as the mean \pm standard error of the mean. Day-by-day comparisons in the MWM were assessed by factorial analysis of variance (ANOVA) with a post hoc test between different groups; oneway ANOVA with Tukey's post hoc test was applied for statistical comparisons. $P<0.05$ was considered statistically significant.

\section{Results}

Inhibition of miR-96 mitigates the learning and memory deficits induced by $\mathrm{CCH}$

We examined the changes in the level of miR-96 in the hippocampus of rats after 2VO surgery. As illustrated in Fig. 1, the level of miR-96 was markedly increased in the 2VO group as compared to the sham group. Antagomir-96 was injected directly into the bilateral hippocampus subfields. After 8 weeks, the level of miR-96 was significantly decreased, as demonstrated by real-time PCR analysis.

The MWM test was used to identify the role of miR-96 in the rat model of $2 \mathrm{VO}$. By analyzing the swimming distance day by day, we found that the $2 \mathrm{VO}$ rats swam a longer distance to the hidden platform than the sham rats, which was reversed by antagomir-96 injection, indicating that the ability to learn to use cues as navigational guides in Antagomir-96 injection rats had improved (Fig. 2A); Day 1: $\mathrm{F}_{(2.15)}=12.817, P=0.001$; Day 2: $\mathrm{F}_{(2,15)}=9.085, P$ $=0.003$; Day 3: $\mathrm{F}_{(2,15)}=5.671, P=0.015 ;$ Day 4: $\mathrm{F}_{(2,15)}=6.445, P=0.01$; Day 5: $\mathrm{F}_{(2,15)}=11.538$, $P=0.001$. As illustrated in Fig. $2 \mathrm{~B}, 2 \mathrm{VO}$ rats had a slower swimming speed day by day as compared with the sham group; however, Antagomir-96 injection reversed this effect; Day 1: $\mathrm{F}_{(2,15)}=3.876, P=0.044 ;$ Day 2: $\mathrm{F}_{(2,15)}=3.982, P=0.041$; Day 3: $\mathrm{F}_{(2,15)}=4.072, P=0.039$; Day $4: \mathrm{F}_{(2,15)}=4.940, P=0.022$; Day $5: \mathrm{F}_{(2,15)}=6.138$, $P=0.011$. The probe trial showed that both platform crossings and time spent in the target quadrant were increased in the Antagomir-96 injection rats as compared with the $2 \mathrm{VO}$ group, indicating that the memory impairment of $2 \mathrm{VO}$ rats was mitigated by miR-96 inhibition (Fig. 2C and 2D). Taken together, these results suggested that inhibition of miR-96 improved the learning and memory abilities of $2 \mathrm{VO}$ rats.

\section{Effect of miR-96 on autophagy under $\mathrm{CCH}$ in} vivo

To identify further the mechanism underlying the effect of miR-96 on the learning and memory deficits induced by $\mathrm{CCH}$, we examined changes of autophagy, which have been reported with both $\mathrm{CCH}$ and miRNAs under different pathophysiological conditions. The number of autophagosomes was increased by $2 \mathrm{VO}$, which was reversed by Antagomir-96 injection (Fig. 3A). Moreover, LC3, a marker of autophagosomes,

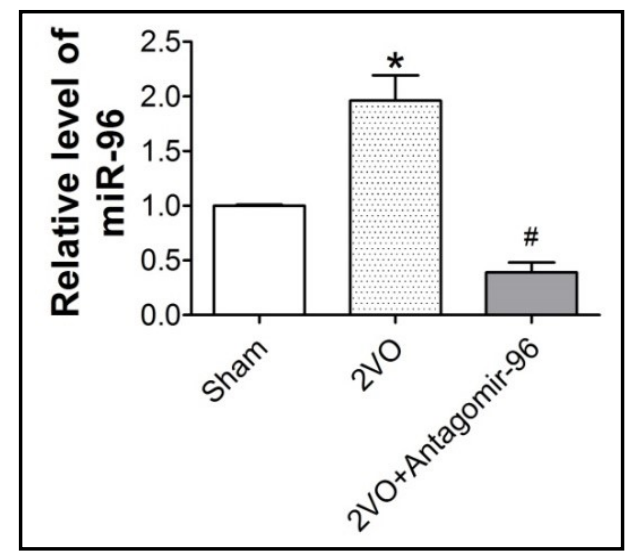

Fig. 1. Level of miR-96 in $2 \mathrm{VO}$ and Antagomir-96 injection rats. The level of miR96 was increased in rats by $2 \mathrm{VO}$ surgery and decreased by Antagomir-96 injection. ${ }^{*} \mathrm{P}<0.05$ vs. sham, ${ }^{"} \mathrm{P}<0.05$ vs. $2 \mathrm{VO}, \mathrm{n}=3$ in each group. 2VO: two-vessel occlusion; Antagomir-96: miR-96 antagomir. 
Liu et al.: Microrna-96 Ameliorates Cognitive Impairment

was significantly increased in the hippocampus of $2 \mathrm{VO}$ rats. In contrast, the level of LC3 was markedly downregulated by Antagomir-96 injection, as demonstrated by western blot and immunofluorescence assays (Fig. 3B and 3C). As shown in Fig. 3D, the level of beclin-1 was also increased in $2 \mathrm{VO}$ rats, which was reversed by Antagomir-96 inhibition, suggesting that inhibition of miR-96 could inactivate autophagy, at least partly, in rats under CCH.

Fig. 2. Effect of miR-96 on cognitive ability after 2VO. (A) Mean daily distance to the hidden platform was examined. ANOVA: Day 1: $\mathrm{F}_{(2,15)}=12.817, \mathrm{P}=0.001$; Day 2: $\mathrm{F}_{(2,15)}=9.085, \mathrm{P}=0.003$; Day 3: $\mathrm{F}_{(2,15)}=5.671, \mathrm{P}=0.015$; Day 4: $\mathrm{F}_{(2,15)}=$ 6.445, $\mathrm{P}=0.01$; Day 5: $\mathrm{F}_{(2,15)}=11.538, \mathrm{P}$ $=0.001 . \mathrm{n}=6$ in each group. (B) Mean daily swimming speed was examined. Day 1: $\mathrm{F}_{(2,15)}=3.876, \mathrm{P}=0.044$; Day 2: $\mathrm{F}_{(2,15)}=3.982, \mathrm{P}=0.041$; Day 3: $\mathrm{F}_{(2,15)}=$ 4.072, $\mathrm{P}=0.039 ;$ Day 4: $\mathrm{F}_{(2,15)}=4.940, \mathrm{P}$ $=0.022$; Day 5: $\mathrm{F}_{(2,15)}=6.138, \mathrm{P}=0.011$, $\mathrm{n}=6$ in each group. (C) Number of times crossing the first quadrant in the probe trial. ${ }^{*} \mathrm{P}<0.05$ vs. sham, ${ }^{*} \mathrm{P}<0.05$ vs. $2 \mathrm{VO}$, $\mathrm{n}=6$ in each group. (D) Percentage time spent in the first quadrant in the probe

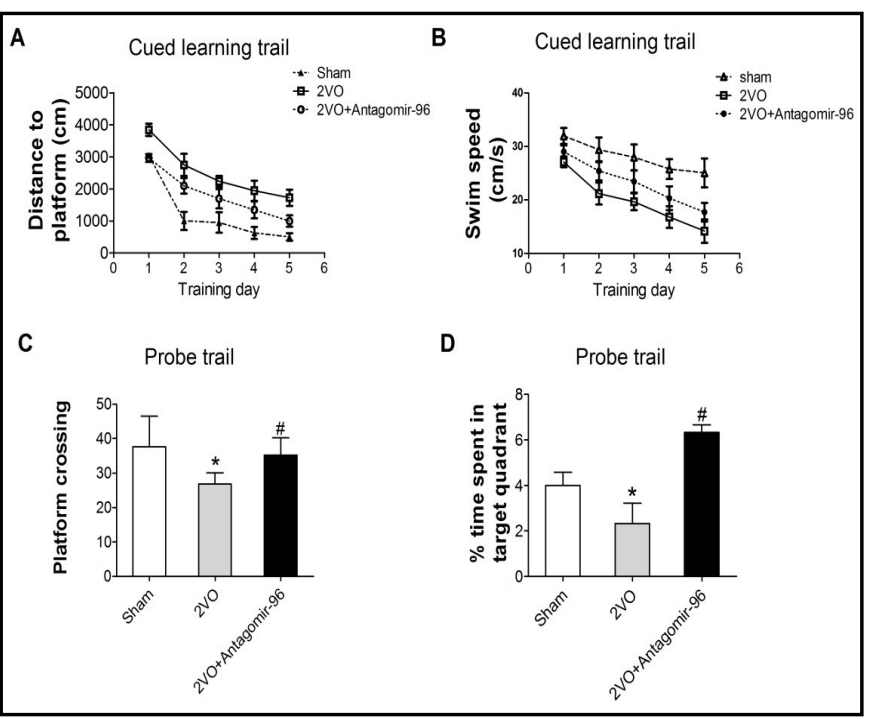
trial. ${ }^{\mathrm{P}}<0.05$ vs. sham, ${ }^{~} \mathrm{P}<0.05$ vs. 2 VO, $\mathrm{n}=6$ in each group. 2 VO: two-vessel occlusion; Antagomir-96: miR96 antagomir.

Fig. 3. Effect of miR-96 on autophagy after 2VO. (A) Electron microscopy analysis of autophagosomes in rats. Scale bars, $20 \mu \mathrm{m}$. (B) The level of LC3 was detected by western blot analysis. Upper panels: representative western blot bands; lower panels: relative digital data analysis. ${ }^{*} \mathrm{P}<0.05$ vs. sham, ${ }^{\#} \mathrm{P}<0.05$ vs. $2 \mathrm{VO}, \mathrm{n}=3$ in each group. (C) Representative staining images with an anti-LC3 (red) antibody in rats of the sham, 2VO, and 2VO+antagomir-96 groups. Scale bars, $10 \mu \mathrm{m}$. (D) The level of beclin-1 was detected by western blot analysis. Upper panels: representative western blot bands; lower panels: relative digital data analysis. ${ }^{*} \mathrm{P}<0.05$ vs. sham, ${ }^{\#} \mathrm{P}<0.05$ vs. $2 \mathrm{VO}, \mathrm{n}=3$ in each group. 2VO: two-vessel occlusion; Antagomir-96: miR-96 antagomir.

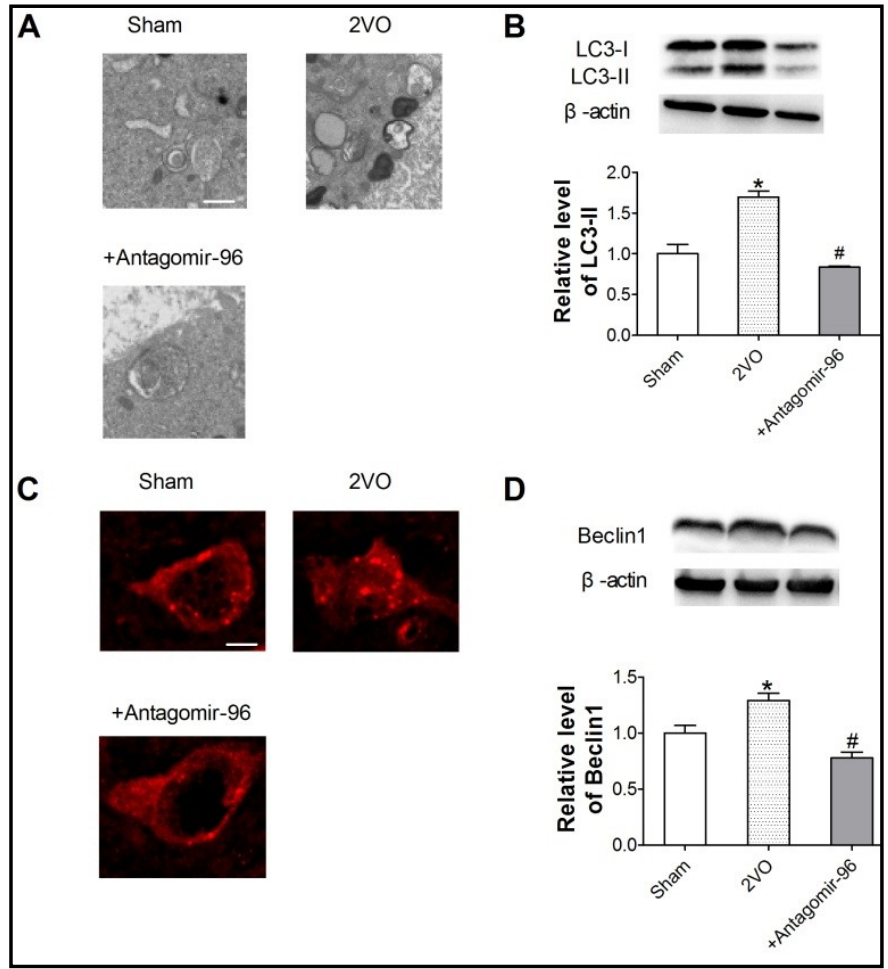


MiR-96 regulates mTOR expression in vivo and in vitro

To assess further the molecular mechanism underlying the effect of miR-96 on autophagy, we detected changes in the level of mTOR, which is involved in $\mathrm{CCH}$ and negatively regulates autophagy by inhibiting downstream ATG genes. As illustrated in Fig. 4, the level of mTOR was reduced by $2 \mathrm{VO}$ and induced by Antagomir-96 injection. Next, we examined whether miR-96 could regulate the expression of mTOR. A miRNA database indicated that the 3'-UTR of mTOR carries binding sites for miR-96, suggesting that mTOR may be a potential target of miR-96 (Fig. 5A). Therefore, the relationship between miR-96 and mTOR was identified by gain- and loss-offunction approaches. As shown in Fig. 5B, miR-96 could inhibit the luciferase activity of mTOR, and this effect was reduced by mutating the miR-96 binding sites. To examine whether changes of miR-96 could affect mTOR expression, miR-96 mimics and Antagomir-96 were transfected into neurons. The successful transfection of miR-96 and Antagomir-96 in neurons was confirmed by real-time PCR (Fig. 5C). As compared with the control group, the protein level of mTOR was downregulated by miR-96 overexpression. Conversely, inhibition of miR-96 by Antagomir-96 upregulated the protein level of mTOR (Fig. 5D), indicating that mTOR is a target of miR-96.

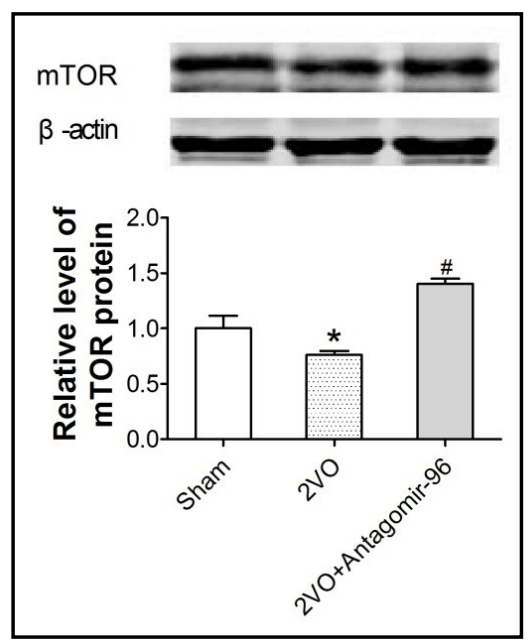

Fig. 4. MiR-96 regulates $\mathrm{mTOR}$ expression in vivo. The level of mTOR was decreased by $2 \mathrm{VO}$ surgery in rats and increased by antagomir-96 injection. ${ }^{*} \mathrm{P}<0.05$ vs. sham, ${ }^{*} \mathrm{P}<0.05$ vs. $2 \mathrm{VO}, \mathrm{n}=3$ in each group. $2 \mathrm{VO}$ : twovessel occlusion; Antagomir-96: miR96 antagomir.

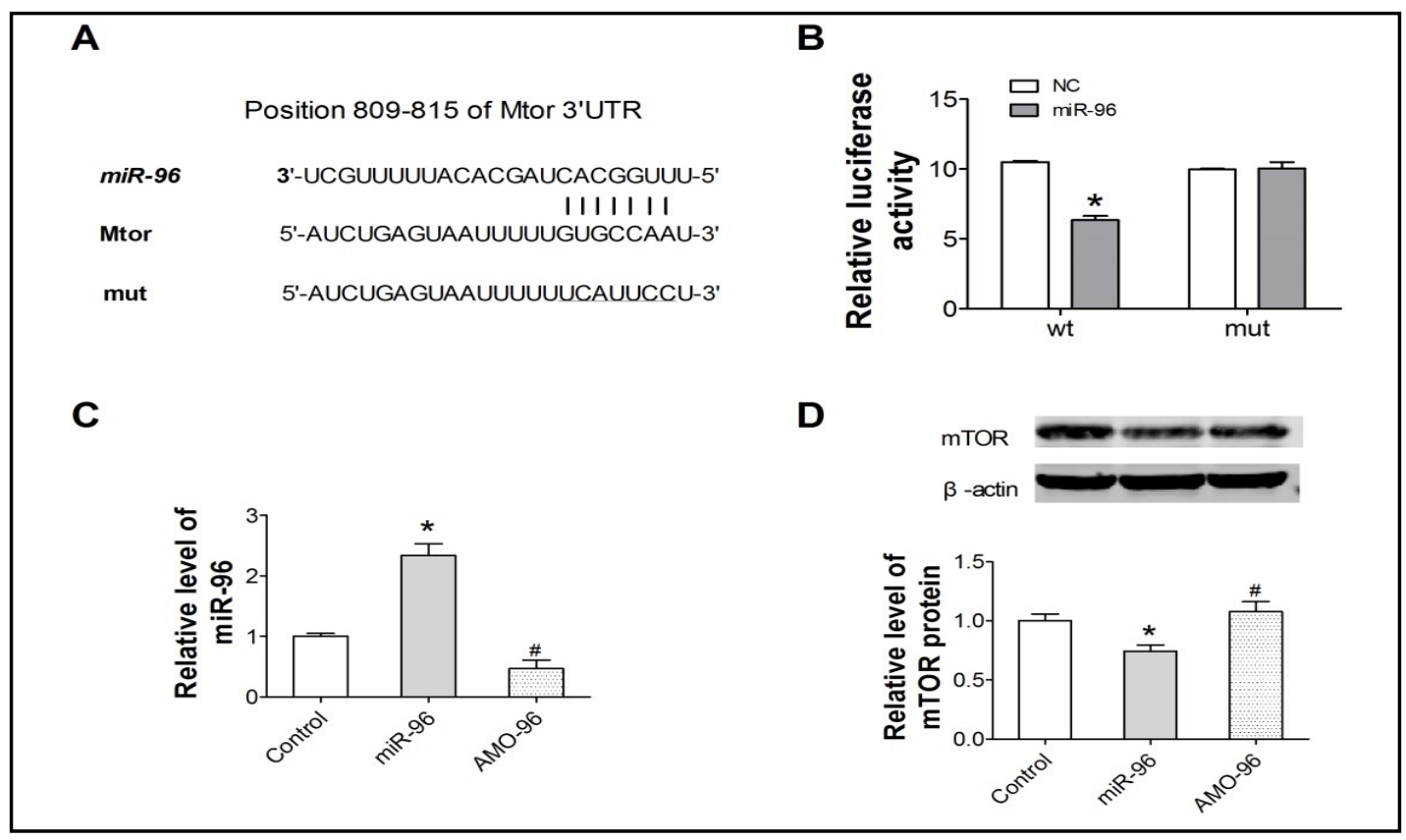

Fig. 5. MiR-96 regulates mTOR expression in vitro. (A) Sequence alignment between the miR-96 seed sequence and the $3^{\prime}$-UTR of mTOR. The mutation sites are underlined. (B) Luciferase reporter assay for interactions between miR-96 and the binding sites or mutation sites in the 3'-UTR of mTOR. (C) Verification of miR-96 uptake by cells. ${ }^{*} \mathrm{P}<0.05$ vs. control. $\mathrm{n}=3$ in each group. (D) Effect of miR-96 on mTOR protein level in cells. ${ }^{*} \mathrm{P}<0.05$ vs. control. $\mathrm{n}=3$ in each group. 


\section{Discussion}

This study showed that the level of miR-96 was increased in rats following CCH. Inhibition of miR-96 ameliorated the cognitive impairment induced by $2 \mathrm{VO}$, as detected by the MWM, and reduced the activation of autophagy, as evidenced by the decreased levels of LC3 and beclin-1 and reduced number of autophagosomes. Furthermore, miR-96 regulated the expression of mTOR in vivo and in vitro. Therefore, this study revealed a novel molecular mechanism of $\mathrm{CCH}$ at the miRNA level and highlighted a potential role for miR-96 as a therapeutic target for $\mathrm{CCH}$.

Autophagy is a lysosomal degradation pathway for the removal of damaged cellular components and plays a critical role in cellular homeostasis, especially in neurons [19, 20]. However, autophagy is a double-edged sword since defective or excessive autophagy may induce cell death [21,22]. Many studies have reported that autophagy is essential for cells in response to fluctuating environments during acute ischemia or $\mathrm{CCH}$. For example, the level of LC3 is increased at 2 or 5 weeks after CCH $[8,9]$. In addition, the levels of LC3 and beclin- 1 and the number of autophagosomes are increased at 8 weeks after 2VO surgery. These observations suggest that autophagy is associated with the pathological process of $\mathrm{CCH}$ and may be a potential target for VaD or AD.

mTOR is a pivotal protein kinase for the regulation of cellular metabolism and can induce neuronal growth and long-term potentiation [23, 24]. Previous studies have shown that mTOR is involved in CCH. For example, L-carnitine enhanced axonal plasticity and ameliorated cognitive impairment by regulating the Pten/Akt/mTOR signaling pathway in rats at 28 days after chronic hypoperfusion [25]. In addition, mTOR is a suppressor of autophagy, and its inhibition is necessary for the initiation of autophagy. However, previous studies have shown that the accumulation of $A \beta$ was increased and $p$-Tau was elevated during $\mathrm{CCH}$. In turn, mTOR signaling activity was decreased and autophagy was impaired due to A $\beta$ accumulation. It is possible that $\mathrm{CCH}$, at least partially, results in a deficiency of autophagy and in turn accelerates the development of $\mathrm{AD}$ or $\mathrm{VaD}$. The present study found that the level of mTOR was decreased in the brain of $2 \mathrm{VO}$ rats at 8 weeks after ligation, which is similar to the finding of a previous report [26].

Recently, miRNAs have been demonstrated to play a critical role in regulating autophagy by modulating the levels of autophagy-related factors. For example, miR-449a could target Bcl-2 to regulate autophagy for inhibiting silica-induced pulmonary fibrosis [27]. MiR-21 could inhibit autophagy and apoptosis induced by hypoxia/reoxygenation injury through the Akt/mTOR signaling pathway [28]. MiR-23b could directly target the 3'-UTR of ATG12 and improve the cognitive impairments induced by traumatic brain injury [29]. The increase in the level of miR-96 in the present study indicates that it could regulate the expression of mTOR in vivo and in vitro. In addition, inhibition of miR-96 could ameliorate the cognitive impairment of rats suffering from $\mathrm{CCH}$.

Taken together, the findings of this study showed that inhibition of miR-96 could regulate the expression of mTOR, inactivate autophagy, and ameliorate cognitive impairment, suggesting a novel mechanism to prevent cognitive impairment due to the autophagy response, indicating that miR-96 may be an endogenous target for $\mathrm{VaD}$ or $\mathrm{AD}$.

\section{Acknowledgements}

This study was funded by National Natural Science Foundation Projects to Dr. Shaohong Fang (No. C080102) and supported by the Key Laboratory of Myocardial Ischemia, Harbin Medical University, Chinese Ministry of Education to Dr. Shaohong Fang (No. KF201203). 


\section{Cellular Physiology Cell Physiol Biochem 2018;49:78-86 \begin{tabular}{ll|l} 
DOI: 10.1159/000492844 & $\begin{array}{l}\text { O 2018 The Author(s). Published by S. Karger AG, Basel } \\
\text { www.karger.com/cpb }\end{array}$ \\
\hline
\end{tabular}}

Liu et al.: Microrna-96 Ameliorates Cognitive Impairment

\section{Disclosure Statement}

The authors declared no conflict of interest.

\section{References}

1 Battistin L, Cagnin A: Vascular cognitive disorder. A biological and clinical overview. Neurochem Res 2010;35:1933-1938.

-2 Xie H, Zhao Y, Zhou Y, Liu L, Liu Y, Wang D, Zhang S, Yang M: MiR-9 Regulates the Expression of BACE1 in Dementia Induced by Chronic Brain Hypoperfusion in Rats. Cell Physiol Biochem 2017;42:1213-1226.

-3 Li YW, Li QY, Wang JH, Xu XL: Contribution of p38 MAPK to the Ameliorating Effect of Enriched Environment on the Cognitive Deficits Induced by Chronic Cerebral Hypoperfusion. Cell Physiol Biochem 2016;40:549-557.

4 Lee KM, Hwang SK, Lee JA: Neuronal autophagy and neurodevelopmental disorders. Exp Neurobiol 2013;22:133-142.

5 Boland B, Nixon RA:Neuronal macroautophagy: from development to degeneration. Mol Aspects Med 2006;27:503-519.

6 Maday S, Holzbaur EL: Autophagosome biogenesis in primary neurons follows an ordered and spatially regulated pathway. Dev Cell 2014;30:71-85.

7 Maday S, Wallace KE, Holzbaur EL: Autophagosomes initiate distally and mature during transport toward the cell soma in primary neurons. J Cell Biol 2012;196:407-417.

-8 Che H, Yan Y, Kang XH, Guo F, Yan ML, Liu HL, Hou X, Liu T, Zong DK, Sun LL, Bao YN, Sun LH, Yang BF, Ai J: MicroRNA-27a Promotes Inefficient Lysosomal Clearance in the Hippocampi of Rats Following Chronic Brain Hypoperfusion. Mol Neurobiol 2017;54:2595-2610.

\$9 Liu L, Li CJ, Lu Y, Zong XG, Luo C, Sun J, Guo LJ: Baclofen mediates neuroprotection on hippocampal CA1 pyramidal cells through the regulation of autophagy under chronic cerebral hypoperfusion. Sci Rep 2015;5:14474.

10 Ai J, Sun LH, Che H, Zhang R, Zhang TZ, Wu WC, Su XL, Chen X, Yang G, Li K, Wang N, Ban T, Bao YN, Guo F, Niu HF, Zhu YL, Zhu XY, Zhao SG, Yang BF; MicroRNA-195 protects against dementia induced by chronic brain hypoperfusion via its anti-amyloidogenic effect in rats. J Neurosci 2013;33:3989-4001.

11 Sun LH, Ban T, Liu CD, Chen QX, Wang X, Yan ML, Hu XL, Su XL, Bao YN, Sun LL, Zhao LJ, Pei SC, Jiang XM, Zong DK, Ai J: Activation of Cdk5/p25 and tau phosphorylation following chronic brain hypoperfusion in rats involves microRNA-195 down-regulation. J Neurochem 2015;134:1139-1151.

12 Sun LH, Yan ML, Hu XL, Peng LW, Che H, Bao YN, Guo F, Liu T, Chen X, Zhang R, Ban T, Wang N, Liu HL, Hou $\mathrm{X}, \mathrm{Ai}$ J: MicroRNA-9 induces defective trafficking of Nav1.1 and Nav1.2 by targeting Nav $\beta 2$ protein coding region in rat with chronic brain hypoperfusion. Mol Neurodegener 2015;10:36.

13 Zhang Y, Liu C, Wang J, Li Q, Ping H, Gao S, Wang P: MiR-299-5p regulates apoptosis through autophagy in neurons and ameliorates cognitive capacity in APPswe/PS1dE9 mice. Sci Rep 2016;6:24566.

14 Lana D, Ugolini F, Melani A, Nosi D, Pedata F, Giovannini MG: The neuron-astrocyte-microglia triad in CA3 after chronic cerebral hypoperfusion in the rat: Protective effect of dipyridamole. Exp Gerontol 2017;96:46-62.

15 Ni J, Ohta H, Matsumoto K, Watanabe H: Progressive cognitive impairment following chronic cerebral hypoperfusion induced by permanent occlusion of bilateral carotid arteries in rats. Brain Res 1994;653:231-236.

-16 Kumaran D, Udayabanu M, Kumar M, Aneja R, Katyal A: Involvement of angiotensin converting enzyme in cerebral hypoperfusion induced anterograde memory impairment and cholinergic dysfunction in rats. Neuroscience 2008;155:626-639.

-17 Mueller SC, Temple V, Oh E, VanRyzin C, Williams A, Cornwell B, Grillon C, Pine DS, Ernst M, Merke DP: Early androgen exposure modulates spatial cognition in congenital adrenal hyperplasia (CAH). Psychoneuroendocrinology 2008;33:973-980.

18 Morris, R., Developments of a water-maze procedure for studying spatial learning in the rat. J Neurosci Methods 1984;11:47-60. 


\section{Cellular Physiology Cell Physiol Biochem 2018;49:78-86 \begin{tabular}{l|l|l} 
DOI: 10.1159/000492844 & $\begin{array}{l}\text { O 2018 The Author(s). Published by S. Karger AG, Basel } \\
\text { www.karger.com/cpb }\end{array}$
\end{tabular}}

Liu et al.: Microrna-96 Ameliorates Cognitive Impairment

19 M'Angale PG, Staveley BE: Inhibition of Atg6 and Pi3K59F autophagy genes in neurons decreases lifespan and locomotor ability in Drosophila melanogaster. Genet Mol Res 2016;15.

20 Yao J, Jia L, Feathers K, Lin C, Khan NW, Klionsky DJ, Ferguson TA, Zacks DN: Autophagy-mediated catabolism of visual transduction proteins prevents retinal degeneration. Autophagy 2016;12:2439-2450.

$>21$ Chen W, Sun Y, Liu K, Sun X: Autophagy: a double-edged sword for neuronal survival after cerebral ischemia. Neural Regen Res 2014;9:1210-1216.

22 Wei K, Wang P, Miao CY: A double-edged sword with therapeutic potential: an updated role of autophagy in ischemic cerebral injury. CNS Neurosci Ther 2012;18:879-886.

-23 Switon K, Kotulska K, Janusz-Kaminska A, Zmorzynska J, Jaworski J: Molecular neurobiology of mTOR. Neuroscience 2017;341:112-153.

24 Swiech L, Perycz M, Malik A, Jaworski J: Role of mTOR in physiology and pathology of the nervous system. Biochim Biophys Acta 2008;1784:116-132.

25 Ueno Y, Koike M, Shimada Y, Shimura H, Hira K, Tanaka R, Uchiyama Y, Hattori N, Urabe T: L-carnitine enhances axonal plasticity and improves white-matter lesions after chronic hypoperfusion in rat brain. J Cereb Blood Flow Metab 2015;35:382-391.

-26 Zhu Y, Zeng Y, Wang X, Ye X: Effect of electroacupuncture on the expression of mTOR and eIF4E in hippocampus of rats with vascular dementia. Neurol Sci 2013;34:1093-1097.

27 Han R, Ji X, Rong R, Li Y, Yao W, Yuan J, Wu Q, Yang J, Yan W, Han L1, Zhu B, Ni C: MiR-449a regulates autophagy to inhibit silica-induced pulmonary fibrosis through targeting Bcl2. J Mol Med (Berl) 2016;94:1267-1279.

28 Huang Z, Wu S, Kong F, Cai X, Ye B, Shan P, Huang W: MicroRNA-21 protects against cardiac hypoxia/ reoxygenation injury by inhibiting excessive autophagy in H9c2 cells via the Akt/mTOR pathway. J Cell Mol Med 2017;21:467-474.

-29 Sun L, Liu A, Zhang J, Ji W, Li Y, Yang X, Wu Z, Guo J: miR-23b improves cognitive impairments in traumatic brain injury by targeting ATG12-mediated neuronal autophagy. Behav Brain Res 2018;340:126-136. 